\title{
Patients' retrospective assessment of palliative chemotherapy for lung or gastrointestinal cancers
}

\author{
Shayan Kassirian, ${ }^{a}$ Gil Schreier, MD, ${ }^{\mathrm{b}}$ and Michael Sanatani, MD
}

${ }^{a}$ Schulich School of Medicine and Dentistry, Western University; ${ }^{b}$ Palliative Care Service, London Health Sciences Centre; and 'Department of Medical Oncology, Schulich School of Medicine and Dentistry, Western University, London, Canada

Background Decision-making about palliative chemotherapy is complex because treatment goals include increased survival, symptom control, and functional improvement.

Objective To examine whether retrospective assessment by chemotherapy-experienced patients could inform decision-making support for future patients.

Methods 51 patients with thoracic or gastrointestinal malignancy, with no further systemic treatment options, completed the Functional Assessment of Chronic Illness Therapy-Treatment Satisfaction (FACIT-TS) survey and answered free-text questions about their past decisions about therapy.

Results FACIT-TS subscale of treatment effectiveness showed $36 \%$ of 49 eligible patients rating effectiveness as being worse than expected, $25 \%$ as expected, $37 \%$ better. $51 \%$ found side effects worse than expected, $19 \%$ as expected, and $28 \%$ better than expected. Textual analysis of survey responses indicated the majority of patients stood by their decision to take chemotherapy but wished they'd had more information about what to expect. Overall, 55\% found chemotherapy to have been worthwhile, $37 \%$ not, $8 \%$ were undecided.

Limitations Accrual was slower than expected, in part because of a lack of awareness by patients that there were no further chemotherapy options available to them. Selection bias may have favoured enrolment from teams open to soliciting patient feedback. Conclusions Although the majority of patients stood by their decisions about palliative chemotherapy based on their understanding of the therapy at the time of making their decisions, there is a discrepancy between initial expectations about chemotherapy and retrospective assessment of chemotherapy effectiveness and side effects. The introduction of end-of-treatment feedback surveys as a routine quality assurance procedure should be considered.

$$
I^{n}
$$
n 2015, 196,000 new cases of cancer were reported in Canada. With rapid advances in cancer care over the past few decades, cancerrelated mortality rates have been dropping steadily. ${ }^{1}$ However, a key modality of cancer treatment that has contributed to this change - chemotherapy can also be a source of distress to many patients. Concern about the side effects of chemotherapy, long waits in the hospital, and the anticipated burden of the therapy on the patient's family may lead to apprehensiveness about it. ${ }^{2}$ Experienced clinicians know how patients - by their own admission are often influenced more by accounts of neighbours' and friends' experiences with chemotherapy than by the oncologist's consultation. This is understandable, because the choice to undergo palliative chemotherapy involves a complex decision process, and patients turn to supports that have aided them in other life situations. It may be difficult for the patient to assess whether the benefits of taking chemotherapy outweigh the possible detrimental effects on an individual level. ${ }^{3}$ The usual approach for the oncologist is to present data from clinical studies to inform patients about expected response rates and adverse events. However, many patients facing palliative chemotherapy would prefer to have more decision aids available to them. As patients have expressed to investigators, insight from current and previous patients would greatly aid this process. Patients have indicated that they would value reports from other patients who had actually taken chemotherapy. For both health care professionals and patients, understanding patient attitudes toward chemotherapy is the first step to better care and treatment decisions.

In some situations, patients may avoid chemotherapy because of their fear of adverse effects. It has also been consistently shown that cancer patients in certain other situations were much more likely

Accepted for publication December 9, 2016. Correspondence: Michael Sanatani, MD; Michael.Sanatani@Lhsc.on.ca Disclosures The authors report no disclosures or conflicts of interest. JCSO 2016;14(12):501-508. @2016 Frontline Medical Communications. doi: 10.12788/icso.0315. 
to approve of treatment with a minimal beneficial effect than were their health care team and other patients who did not have cancer. ${ }^{4,5,6}$ Attitudes varied considerably for different chemotherapy regimens, treatment intents, and different cancers. One study reported that breast cancer patients who have previously experienced treatment had a significantly more positive outlook on the effect of adjuvant chemotherapy compared with patients who had not had cancer. ${ }^{7}$ There are situations, however, where evidence supports not giving chemotherapy. For example, a guide published by the American Society for Clinical Oncology indicates that chemotherapy should not be used for solid tumor patients with a low performance status and no benefit from prior treatment. ${ }^{8}$ One study of patients with various types of advanced-stage cancer, found that palliative chemotherapy improved quality of life overall. However, it had no effects on quality of life near death. ${ }^{9}$ Mayrbäurl and colleagues studied advanced colorectal cancer patients and noted that although quality of life actually stabilized during the first- and second-line chemotherapy, it decreased steadily with initiation of third-line chemotherapy. ${ }^{10}$ There is very little feedback data available on individual patients' assessments of their palliative-intent chemotherapy. Previous studies have evaluated the use of palliative chemotherapy, including analyzing efficacy, effect on quality of life, and patient attitudes toward it in a prospective fashion. Palliative chemotherapy can certainly prolong survival and improve quality of life for patients with cancers such as colorectal and ovarian cancers. ${ }^{11,12}$ Patients' assessment of that benefit on an individual basis after experiencing the treatment is not known. We hypothesized that exploring the retrospective vantage point may offer assessments of the key question that patients face, namely whether taking palliative chemotherapy is worthwhile or not.

We gathered the retrospective insights of palliative chemotherapy patients about their treatment. Such feedback from patients may provide valuable insight to help guide future patients through their chemotherapy decision-making process. In addition to using the Functional Assessment of Chronic Illness Therapy-Treatment Satisfaction (FACIT-TS) survey, a validated questionnaire on treatment satisfaction, we used open-ended questions to gather patient opinions as free-text responses that were subjected to qualitative thematic analysis.

\section{Methods}

Patients were approached by members of the study team in outpatient clinics, on the ward, or during palliative care home visits about completing the survey to find out about their views on having taken palliative-intent chemotherapy. In cases in which the treating oncologist was a study team member, patients' surveys were collected from the patients directly by the research coordinator or nurse after they were filled out. This was done so the patients could feel free to write their true opinion about the treatment provided by the oncology team, and they were reassured about confidentiality and anonymisation of the collected data. The study was approved by the Human Subjects Research Ethics Board of Western University.

\section{Eligibility}

Eligible patients had received at least 1 cycle of palliativeintent systemic cytotoxic chemotherapy for a gastrointestinal, thoracic, or primary unknown malignancy. No further systemic chemotherapy was to be planned after this survey, and all patients were to be aware of that fact. Patients who declined further therapy and those for whom no further chemotherapy was being offered by the oncology team were eligible. Patients with plans for future radiotherapy were eligible.

Patients with delirium, dementia or cognitive impairment as diagnosed by the treating physician, brain metastases, hepatic encephalopathy, or decreased level of consciousness that in the opinion of the study team would make survey answers unreliable were excluded. Patients who had at any time in their cancer treatment taken part in a clinical treatment trial were also excluded. Clinical trial patients were excluded because our survey was aimed at elucidating opinions about chemotherapy taken for one's own health, rather than treatment within a trial framework where a patient may find it to have been meaningful or worthwhile purely by virtue of the perception of having contributed to science and the care of future patients.

\section{Survey}

The questionnaire consisted of two components: the FACIT-TS, and a treatment decisions retrospective survey with open-ended questions designed for the purposes of this study. The questions on the treatment decisions retrospective survey were:

1. Knowing what you do now about your illness and all the treatments you received for your cancer, are there any past decisions you would have made differently?

2. What could the health care team have done better to help you make the right decisions at the beginning?

3. How did chemotherapy help you?

4. What was the worst thing about taking chemotherapy?

5. Overall, do you think taking any chemotherapy to control the cancer was worthwhile?

FACIT-TS responses were scored according to the validated method. ${ }^{13}$ Responses to the aforementioned questions 1 through 5 on the treatment decisions survey were transcribed verbatim to an anonymized database and analyzed using qualitative thematic analysis. In addition, correlations between a positive response to question 5 about chemotherapy being worthwhile and baseline characteristics were tested in an exploratory fashion. 


\section{Results}

During December 2010 and December 2015, 51 patients were approached for participation in the survey at the London Regional Cancer Program outpatient clinics, the palliative care ward at London Health Sciences Centre, and by the home-visiting palliative care team. All of the patients who were approached agreed to participate, however, one patient did not sign the consent form before he died, and one patient was ineligible because he had received only curative-intent chemotherapy, resulting in 49 evaluable surveys (Table).

\section{FACIT-TS}

The FACIT-TS questionnaire assesses satisfaction with treatment. Responses to questions 1 and 2 are presented in Figure 1. Questions 4 (Did you feel you received the treatment that was right for you?) and 5 (Are you satisfied with the effects of this treatment so far?) are combined to form a Treatment Satisfaction Subscale (0-100, higher number indicates higher satisfaction). For the 46 patients who completed those questions, the mean result was 54 (SD, 33). Questions 6 (Would you recommend this treatment to others with your illness?) and 7 (Would you choose this treatment again?) are combined to form the Recommendation subscale. For the 48 patients who completed those questions, the mean result was $58(\mathrm{SD}, 40)$.

\section{Treatment decisions retrospective survey}

Patient responses to questions were qualitatively analyzed (Figures 2-5).

Question 1: Knowing what you do now about your illness and all the treatments you received for your cancer, are there any past treatment decisions you would have made differently?

The majority of patients (33 of 49,68\%) responded that they would not make any treatment decisions differently, mostly with a straight No response. Other responses indicated that the health care team knew which treatment decisions were best or that they would try chemotherapy first anyways. One patient wished that they could continue chemotherapy despite contraindications with other health concerns. Sixteen patients found their treatment plan unfavourable. There were patients who wanted alternatives to chemotherapy, such as more radiation, more targeted therapies, or looking into alternative medicine. There were also patients who wished they had not taken chemotherapy, with one saying that they would prefer to enjoy their remaining life without chemotherapy. Another would have preferred that the chemotherapy length was extended and the dosage lowered to reduce the side effects.

There were also patients who did not have an unfavourable view of their treatment plan, but had some suggestions about what they would do differently. The responses indicated earlier initiation of chemotherapy and, when appro-

\begin{tabular}{|c|c|}
\hline Characteristic & Value \\
\hline No. of men/women & $28 / 21$ \\
\hline Mean age, y (range) & $68(38-88)$ \\
\hline $\begin{array}{l}\text { Median no. of cycles of palliative chemo- } \\
\text { therapy (range) }\end{array}$ & $4(1-26)$ \\
\hline $\begin{array}{l}\text { Median no. of different regimens of pallia- } \\
\text { tive chemotherapy (range) }\end{array}$ & $1(1-6)$ \\
\hline \multicolumn{2}{|l|}{ Primary tumor } \\
\hline Lung & 17 \\
\hline Gastro-esophageal & 6 \\
\hline Pancreatic & 7 \\
\hline Colorectal/small bowel & 14 \\
\hline Neuroendocrine (GI) & 1 \\
\hline Primary unknown (categorized as Gl) & 3 \\
\hline Radiation received & 26 \\
\hline
\end{tabular}

priate, palliative care as well as more careful self-observation of signs and symptoms.

\section{Question 2: What could the health care team have done better to} help you make the right treatment decisions at the beginning?

The themes in the responses to this question were generally very clear. If there was anything the health care team could do to help, it would be by improving patient autonomy, for example by giving more information about the treatments and explaining alternatives more thoroughly. More attention to the patients' needs and more flexibility and patient say on whether to continue treatment were suggested. Patients also would have appreciated being given copies of the studies used in decision making, and would have liked to have been put in contact with other cancer patients to improve their knowledge about their own condition. Otherwise, patients praised the health care team for being supportive, dedicated, and trying their best relative to the situation.

\section{Question 3: How did chemotherapy help you?}

The positive responses generally followed the intended purposes of chemotherapy in that it was felt to have directly controlled the cancer, but also had other health benefits, both psychological and physical. The chemotherapy gave hope, extended life, and relieved cancer symptoms. One patient also noted that oral chemotherapy was less intrusive in their life, and therefore had psychological benefits. However, a significant proportion of responses (24 of 50, 48\%) indicated the chemotherapy was unsuccessful or had no perceivable benefit, or was even detrimental. 

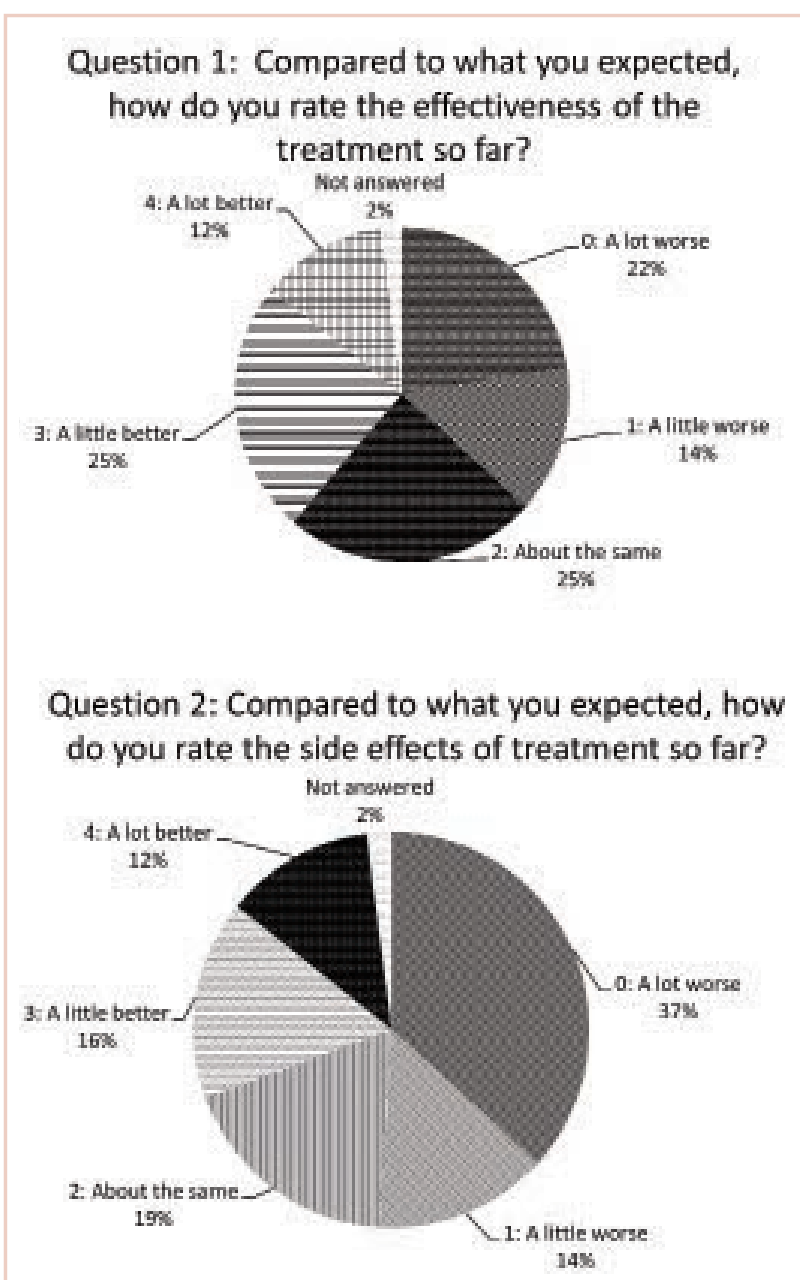

FIGURE 1 Responses to questions 1 and 2 of the Functional Assessment of Chronic Illness Therapy-Treatment Satisfaction questionnaire

\section{Question 4: What was the worst thing about taking chemotherapy?}

Patient responses to question 4 covered a range of chemotherapy effects. The various side effects of chemotherapy were present in many responses. Direct results of treatment administration, such as repeated needle usage, were also cited. Many of the responses indicated psychological, rather than physical, effects. Some patients felt lonely, scared, or on edge because of the anticipation of side effects. Taking pills and having to wait in the chemotherapy suite during treatment also interfered with their daily lives. Finally, certain patients felt "lost" because of a lack of resources or family support.

Question 5: Overall, do you think taking any chemotherapy to control the cancer was worthwhile? (Yes/No)

Of the 49 respondents, 27 (55\%) answered Yes, and 22 $(45 \%)$ answered No $(18,37 \%)$ or were undecided $(4,8 \%)$.
In an exploratory analysis of the influence of age, gender, tumour site (thoracic and gastrointestinal), or number of cycles of palliative chemotherapy received, using a Wilcoxon 2-sample test the only significant association was that patients who found chemotherapy worthwhile had received more cycles of palliative chemotherapy than did those who had not (mean, 7 vs 3 cycles, $P<.001$ ).

\section{Discussion}

In this study, we asked patients who had completed all palliative chemotherapy for their feedback about the experience of taking such treatment. During the conduct of the study, several interesting observations were made. Although our study met with support from the medical oncologists at our center, patient accrual took longer than expected. Several factors may account for this. Screening for the study depended on the oncologist or nurse remembering to ask patients about their willingness to take the survey after the end of chemotherapy, a point in time where possibly very emotionally difficult discussions take place. Even if the patient's condition was stable and the final chemotherapy dose was already several weeks in the past, enrolment was often difficult because of the inclusion criterion that patients had to be aware there were no further chemotherapy options. We found that oncologists' documentation often did not articulate this clearly, for example not wanting to rule out the possibility of future treatments or trials, or deferring the end of treatment discussion to be addressed by a palliative care clinic. At the same time, outpatient palliative care physicians often left discussion of the end of treatment to the medical oncologist, so they were not willing to enroll patients in the study unless the patient endorsed that the goal of treatment was exclusively supportive going forward. The palliative care team also expressed concern about awakening regrets while the patient was on their deathbed. This suggests an issue of a biased view by health care teams of assuming regret is highly likely in regard to chemotherapy. Finally, we discovered significant heterogeneity among oncologists in terms of follow-up after the end of systemic treatment, with many oncologists not giving any follow-up after completion of treatment and referring back to the family physician immediately. These patients, although then clearly aware that the end of treatment had been reached, were lost to our study enrolment. These observations highlighted the fact that at our institution, patient care at the end of systemic treatment is rather heterogeneous and unstructured, and has led to renewed dialogue between the palliative care and oncology services about ways to have a more standardized, supportive guidance approach for patients at this point in their illness.

A major recurring theme in this study was that the patients seem unprepared for, and even disappointed with, the effectiveness of their treatment (Figure 1). Although a 


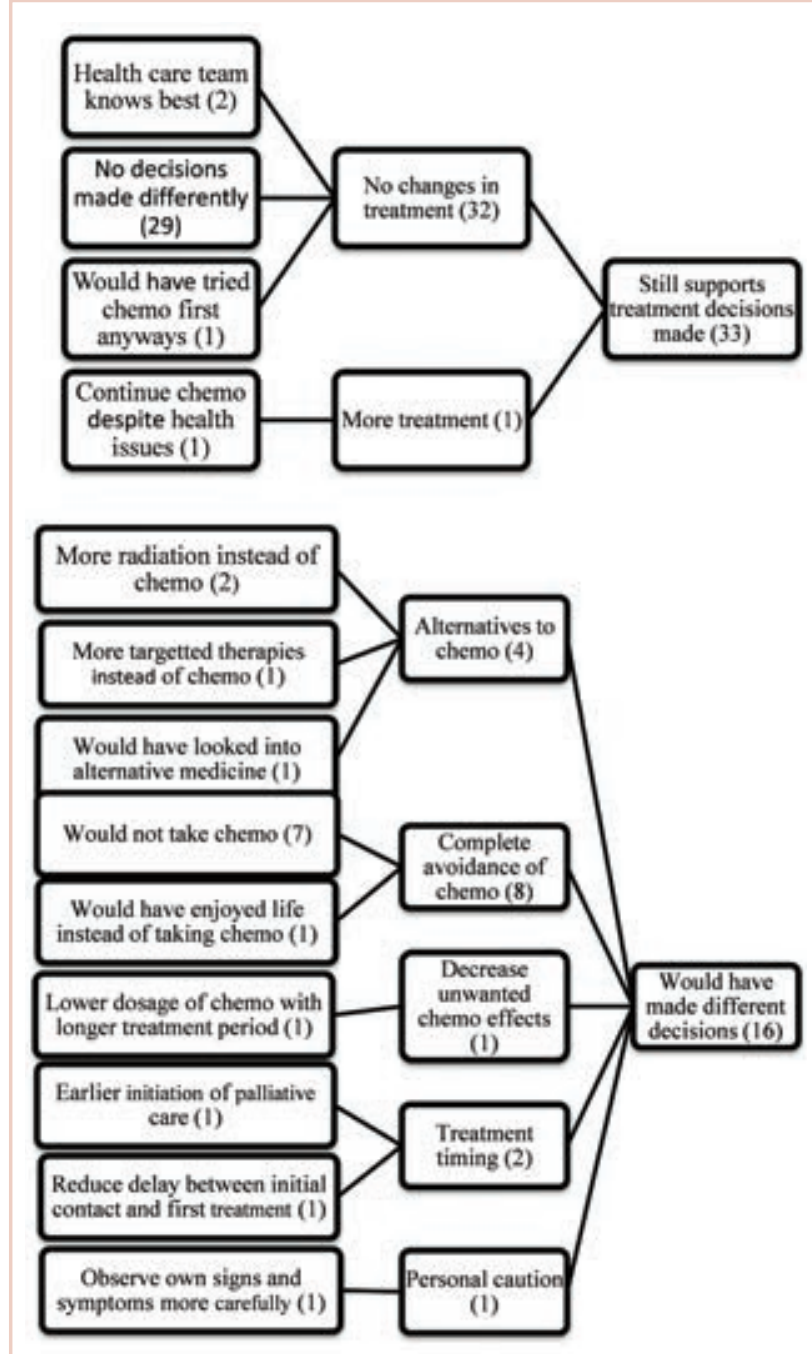

FIGURE 2 Themes found in Question 1 responses in the Treatment Decisions Retrospective Survey: Knowing what you do now about your illness and all the treatments you received for your cancer, are there any past treatment decisions you would have made differently? Note the networks present specific themes towards the left side and broader themes toward the right side. The numbers in boxes indicate how many patient comments fall under that theme. These numbers do not necessarily add up to 49 patients since a single comment may cover multiple themes or some comments do not provide meaningful information.

large proportion had their expectations match reality, the rest were divided between outcomes that exceeded expectations and those that did not meet expectations. Figure 4 also shows that patient opinions were divided evenly on whether or not they felt that their chemotherapy was beneficial. Judging by these two figures, it would seem there is a significant discrepancy between the patients' a priori expectations of treatment effectiveness and the effectiveness perceived retrospectively. It is also important to note that there was a positive correlation between the number of chemotherapy cycles received and patient satisfaction.

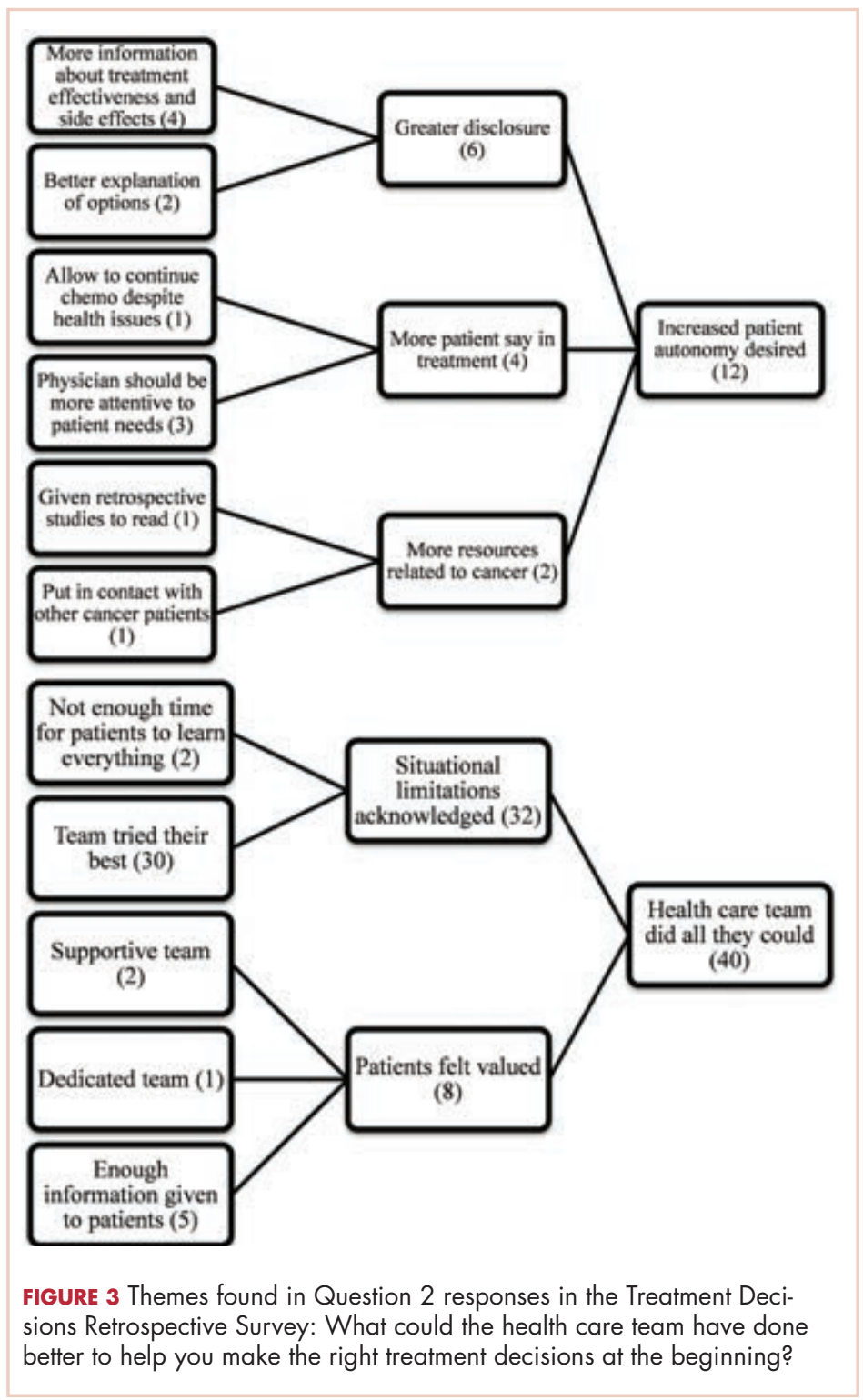

Chemotherapy is prescribed while it is still effective, so patients having more cycles of chemotherapy would generally be more satisfied as the positive treatment effect as assessed by the oncologist would have been ongoing. Inversely, patients with a brief or lack of clinical response would receive less chemotherapy and in our study were less satisfied.

In terms of side effects, there again seems to be a theme of patient unpreparedness in regard to the rigours of undergoing chemotherapy. Figure 1 shows that the majority of patients found that the side effects were worse than they expected. As shown in Figure 5, the majority of patients cited physical side effects as the worst aspect of taking chemotherapy, although there were also several psychological concerns. Being unprepared for these side effects may have manifested itself as fear or anxiety, which 


\section{Original Report}

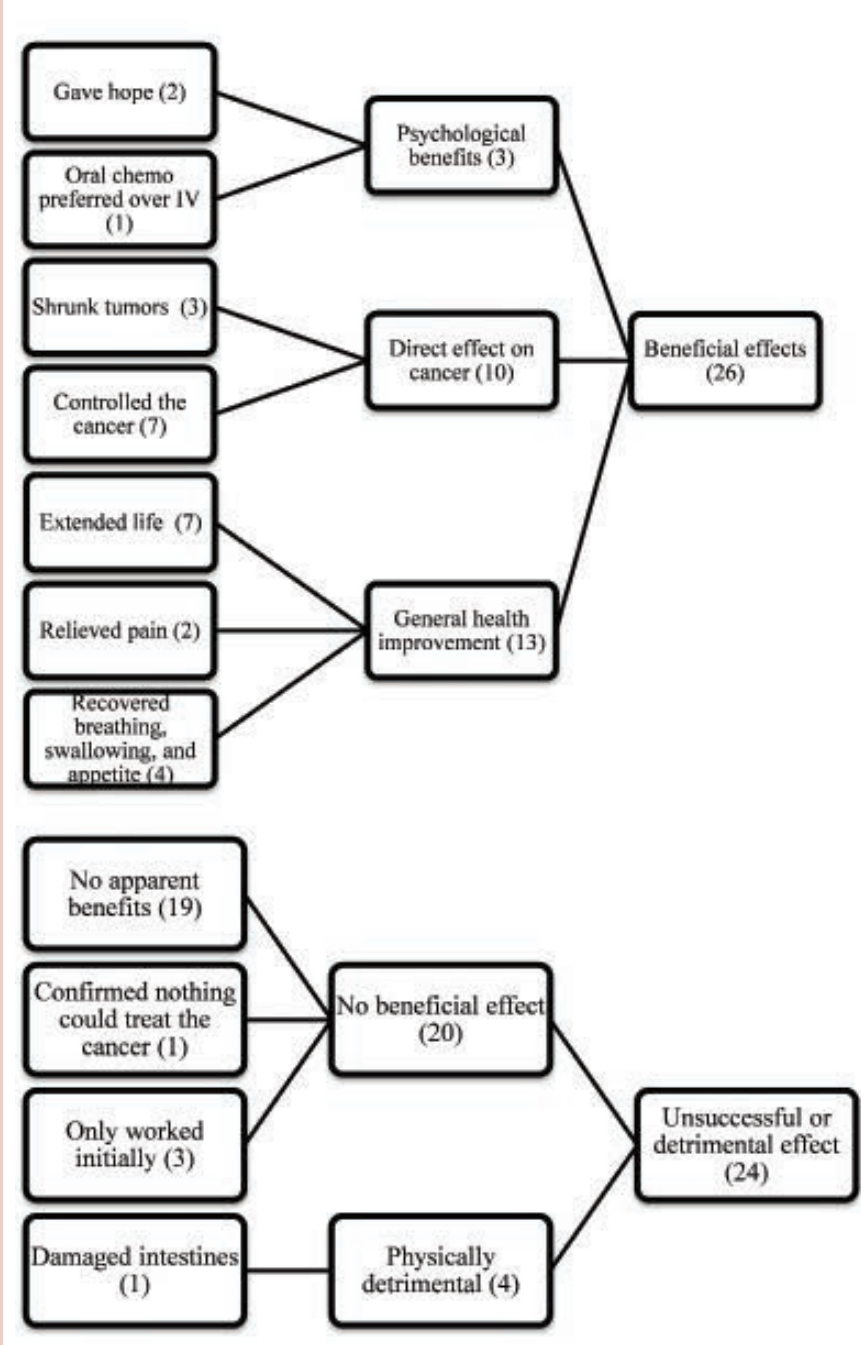

FIGURE 4 Themes found in Question 3 responses in the Treatment Decisions Retrospective Survey: How did chemotherapy help you?

may have exacerbated any negative impact of chemotherapy on the patients' quality of life. That this impression remained so important to patients recalling their experience even after many months of treatment, suggests that it is critical to attend to patients' expectations proactively, rather than offering support in response to toxicity or progression after the fact.

Many patients felt they were unprepared for both the treatment effectiveness and its side effects, so the issue would seem to lie with lack of information or education. Specifically, one patient's response to question 2 of the retrospective survey was that they would like more information, explanations, and resources about their cancer and treatment (Figure 3). Figure 5 also shows that lack of knowledge about what was happening with their cancer was one of the worst things about the chemotherapy. Having this information at an earlier stage would help match patients' expectations to reality to a greater extent,

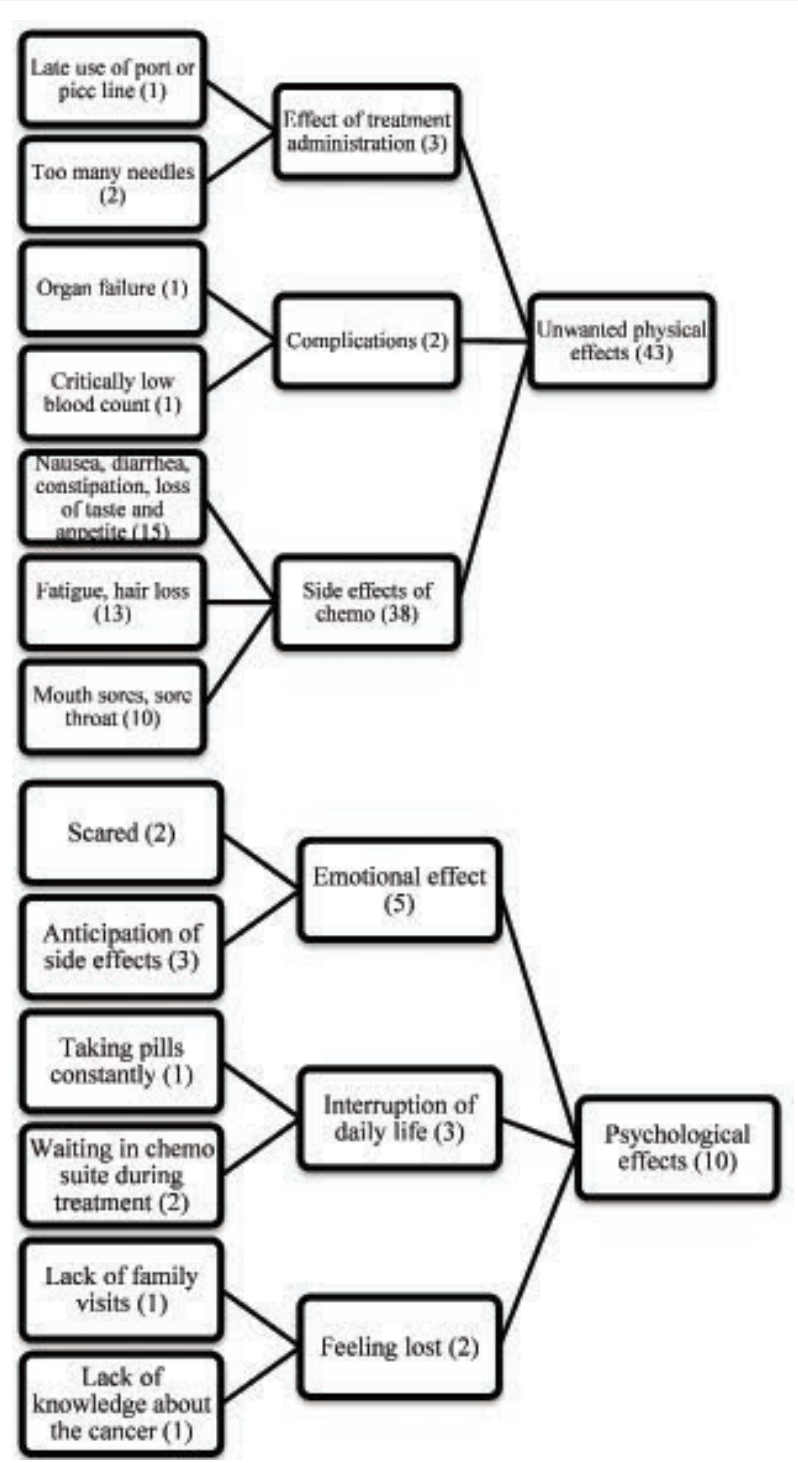

FIGURE 5 Themes found in Question 4 responses in the Treatment Decisions Retrospective Survey: What was the worst thing about taking chemotherapy?

and thus improve patient satisfaction and, by that token, quality of life.

Although physicians and nurses generally make a valiant effort to fulfill disclosure requirements through consultations, phone calls, and brochures, it still does not seem to be sufficient to prepare patients as much as they would like to be prepared. As Harden points out in regard to a different educational setting (medical trainees), education consists not just of providing information but also of verifying that the content has been learned. ${ }^{14}$

Our results confirm the need addressed in the cancer patient education literature to develop ways to prepare patients for an experience completely new for them, in a situation of high emotional stress and information over- 
load. A promising approach is outlined by Pearce and colleagues ${ }^{15}$ with the PRISMS taxonomy for self-management support in long-term conditions, which may lend itself to adaptation to the cancer chemotherapy environment and which also includes skills education beyond knowledge acquisition. Rigdon ${ }^{16}$ has outlined educational strategies in older cancer patients, with a strong focus on assessment of learner retention of information. Jones and colleagues ${ }^{17}$ have developed a course for oncology professionals aimed at improving patient preparation for chemotherapy. However, even with excellent and effective patient education and preparation, one persistent difficulty is that it is often not possible to predict what a given cancer patient will experience. Efforts are underway by the oncology community in general to improve prediction of adverse events from chemotherapy and to individualize treatments. ${ }^{18,19} \mathrm{In}$ addition, counselling strategies that focus specifically on supporting patients in their readiness to face, and ability to cope with, the unpredictability and uncertainty of their treatments and future. In follow-up to our study, we started an initiative to improve communication in serious illness, based in part on the approach developed by Bernacki and colleagues. ${ }^{20}$ Both medical and radiation oncology postgraduate trainees have received training regarding in-depth exploration of patients' views of the future, and this communication initiative will be expanded to attending physicians as well. We envision a future study of patient retrospective perspectives to assess the overall impact of such an intervention on patient satisfaction regarding their treatments received.

Our study has several significant limitations. For reasons we have already described, we had a small sample size and within that, a significant heterogeneity of chemotherapy regimens used. To increase the generalizability of our results and be able to make more robust inferences about the administration of specific regimens and patient satisfaction within tumour subgroups, a larger sample would be needed. However, as each patient's treatment course is unique in structure and duration (a well-known fact also highlighted by our study), subgroups would have to remain fairly specific, again diminishing generalizability to the overall oncology patient population. Another limitation to the generalizability of our study was selection bias, possibly introduced into the current data by patients' lack of clarity about goals of care in some cases and rapid discharge after treatment in other cases. Our study may be biased toward more respondents being patients followed by oncology care teams who clearly articulate goals of care, follow patients even after the end of antineoplastic treatment, and are open to soliciting feedback from patients. It remains to be seen how the results would be different if all cancer clinic patients were asked about their thoughts on their past chemotherapy, a study design that would likely require introduction of satisfaction sur- veys as a routine quality control measure once no further chemotherapy orders are entered for a given patient. In fact, presenting patients with such a survey as a matter of routine when the oncologist orders discontinuation of all systemic therapy through the electronic order entry system will be considered at our center.

\section{Conclusion}

Overall, our results show a significant discrepancy between patients' expectations and actual experience with palliative chemotherapy, an impression that persists even past the end of all palliative systemic therapy. This, in turn, may negatively affect patient satisfaction. Patient responses to the retrospective survey suggested a few common and significant themes: patients usually stand by their decision to pursue palliative chemotherapy, they would like more information and disclosure about the therapy, and the treatment has significant psychological and emotional effects. Also, openly discussing and accepting the end of palliative chemotherapy is a very sensitive and fearful subject not only for patients, but for their health care teams, leading to possibility of selection bias in the current study. Preparing patients earlier on in the treatment trajectory for this inevitable situation may allow the end of treatment to be discussed more easily and may facilitate collection of more robust feedback data from a broad spectrum of oncology and palliative care teams. Our study adds patient perspectives from a retrospective, end-oftreatment vantage point to the existing body of knowledge derived mainly from shorter-term assessments.

\section{Practice implications}

Research into more individualized prediction of chemotherapy adverse events, as well as support regarding dealing with uncertainty may improve patient satisfaction with having taken chemotherapy. More routine and clear articulation of the goals of care will facilitate future quality assurance and exploratory research around patient education and decision-making. Our study confirms a strong need for the development and implementation of more specific and effective chemotherapy-related patient educational tools and strategies at our centre, as well as a need to support patients in facing uncertainty in general, in order to better prepare the patients for the experience they are facing. Although limited in its generalizability, our study highlights the fact that even as some patients approach the end of their life after many months of treatment, their overall assessment of their palliative systemic treatment is still coloured by their recollection of their preparedness for what they experienced. Enhanced communication initiatives aimed at exploring patient expectations, introduction of routine feedback at the end of systemic treatment, and a well-structured care process at that time may be key to patient satisfaction during systemic treatment and beyond. 


\section{Acknowledgments}

The authors thank Larry Stitt of the Department of Epidemiology and Biostatistics, Western University, for statistical analysis, and Francis Whiston, Clinical Research Unit, London Regional Cancer Program,

\section{References}

1. Canadian Cancer Statistics publication - Canadian Cancer Society. http://www.cancer.ca/en/cancer-information/cancer-101/canadiancancer-statistics-publication/?region=bc. Published October 2016. Accessed December 9, 2016.

2. Carelle N, et al. Changing patient perceptions of the side effects of cancer chemotherapy. Cancer. 2002;95:155-163.

3. Matsuyama R, Reddy S, Smith TJ. Why do patients choose chemotherapy near the end of life? A review of the perspective of those facing death from cancer. J Clin Oncol. 2006;24:3490-3496.

4. Payne S A. A study of quality of life in cancer patients receiving palliative chemotherapy. Soc Sci Med. 1992;35:1505-1509.

5. Slevin ML, et al. Attitudes to chemotherapy: comparing views of patients with cancer with those of doctors, nurses, and general public. BMJ. 1990;300:1458-1460.

6. Bremnes RM, Andersen K, Wist EA. Cancer patients, doctors and nurses vary in their willingness to undertake cancer chemotherapy. Eur J Cancer. 1995;31:1955-1959.

7. Jansen S, et al. Explaining differences in attitude toward adjuvant chemotherapy between experienced and inexperienced breast cancer patients. J Clin Oncol. 2005;23:6623-6630.

8. Schnipper LE, et al. American Society of Clinical Oncology identifies five key opportunities to improve care and reduce costs: the top five list for oncology. J Clin Oncol. 2012;30:1715-1724.

9. Prigerson HG, Bao Y, Shah MA, et al. Chemotherapy use, performance status, and quality of life at the end of life. JAMA. Oncol 2015;1:778-784

10. Mayrbäurl B, et al. Quality of life across chemotherapy lines in patients with advanced colorectal cancer: a prospective single-center observational study. Support Care Cancer. 2015;24:667-674.

11. Simmonds P C. Palliative chemotherapy for advanced colorec-
London Health Sciences Centre, for database setup and management. We also thank Kylea Potvin, of the Department of Medical Oncology, Western University, for guidance on enhanced communication initiatives.

tal cancer: Systematic review and meta-analysis. Br Med J Int Ed. 2000;321:531-535.

12. Tsubamoto $H$, et al. Benefit of palliative chemotherapy and hospice enrollment in late-stage ovarian cancer patients. J Obstet Gynaecol Res. 2014;40:1399-1406.

13. Peipert JD, et al. Development and validation of the functional assessment of chronic illness therapy treatment satisfaction (FACIT TS) measures. Qual Life Res. 2014;23:815-24.

14. Harden RM, Laidlaw JM. Essential skills for a medical teacher. Churchill Livingstone, Toronto, 2012.

15. Rigdon A S. Development of patient education for older adults receiving chemotherapy. Clin J Oncol Nurs. 2010;14:433-41.

16. Pearce G, et al. The PRISMS taxonomy of self-management support: derivation of a novel taxonomy and initial testing of its utility. J Health Serv Res Policy. 2016;21:73-82.

17. Jones JM, et al. Maximizing your patient education skills (MPES): a multi-site evaluation of an innovative patient education skills training course for oncology health care professionals. Patient Educ Couns. 2011;84:176-184.

18. Dranitsaris G, Lacouture ME. Development of prediction tools for diarrhea and rash in breast cancer patients receiving lapatinib in combination with capecitabine. Breast Cancer Res Treat. 2014;147:631-638.

19. Miller FA, et al. Testing personalized medicine: patient and physician expectations of next-generation genomic sequencing in late-stage cancer care. Eur J Hum Genet. 2014; 22:391-395.

20. Bernacki R, Hutchings M, Vick J, et al. Development of the serious illness care program: a randomised controlled trial of a palliative care communication intervention. BMJ Open. 2015;5:1-15 doi: 10.1136/ bmjopen-2015-009032. 